\title{
Article \\ Exploring Early Pre-Symptomatic Detection of Influenza Using Continuous Monitoring of Advanced Physiological Parameters during a Randomized Controlled Trial
}

\author{
Nir Goldstein 1,2, + , Arik Eisenkraft $2,3, *,+\mathbb{D}$, Carlos J. Arguello ${ }^{4}$, Ge Justin Yang ${ }^{5}$, Efrat Sand ${ }^{2}$, Arik Ben Ishay ${ }^{2}$, \\ Roei Merin ${ }^{2}$, Meir Fons ${ }^{2} \mathbb{D}$, Romi Littman ${ }^{2}$, Dean Nachman ${ }^{3,6}$ and Yftach Gepner ${ }^{1} \mathbb{D}$
}

1 Department of Epidemiology and Preventive Medicine, School of Public Health, Sackler Faculty of Medicine, and Sylvan Adams Sports Institute, Tel-Aviv University, Tel-Aviv 6997801, Israel; nir@bio-beat.com (N.G.); gepner@tauex.tau.ac.il (Y.G.)

2 Biobeat Technologies LTD, Petah Tikva 4951122, Israel; sand@bio-beat.com (E.S.); arik@bio-beat.com (A.B.I.); m.roei@bio-beat.cloud (R.M.); meir@bio-beat.com (M.F.); romi@bio-beat.cloud (R.L.)

3 The Institute for Research in Military Medicine, The Hebrew University Faculty of Medicine, The Israel Defense Force Medical Corps, Jerusalem 9112102, Israel; deannahman@gmail.com

4 Leidos, Reston, VA 20190, USA; Carlos.ArguelloOrtiz@hhs.gov

check for

updates

Citation: Goldstein, N.;

Eisenkraft, A.; Arguello, C.J.;

Yang, G.J.; Sand, E.; Ishay, A.B.;

Merin, R.; Fons, M.; Littman, R.;

Nachman, D.; et al. Exploring Early

Pre-Symptomatic Detection of

Influenza Using Continuous

Monitoring of Advanced

Physiological Parameters during a

Randomized Controlled Trial. J. Clin.

Med. 2021, 10, 5202. https://

doi.org/10.3390/jcm10215202

Academic Editors: Alessandra Falchi,

Giuseppe Signoriello and

Nicola Petrosillo

Received: 2 September 2021

Accepted: 5 November 2021

Published: 8 November 2021

Publisher's Note: MDPI stays neutral with regard to jurisdictional claims in published maps and institutional affiliations.

Copyright: (c) 2021 by the authors. Licensee MDPI, Basel, Switzerland. This article is an open access article distributed under the terms and conditions of the Creative Commons Attribution (CC BY) license (https:/ / creativecommons.org/licenses/by/ $4.0 /)$.
5 Department of Health and Human Services, Biomedical Advanced Research and Development Authority (BARDA), Washington, DC 20201, USA; Ge.Yang@hhs.gov

6 Heart Institute, Hadassah Medical Center, The Hebrew University of Jerusalem, Jerusalem 9112102, Israel

* Correspondence: aizenkra@gmail.com; Tel.: +972-52-9210-896

+ Equal contribution.

\begin{abstract}
Early detection of influenza may improve responses against outbreaks. This study was part of a clinical study assessing the efficacy of a novel influenza vaccine, aiming to discover distinct, highly predictive patterns of pre-symptomatic illness based on changes in advanced physiological parameters using a novel wearable sensor. Participants were frequently monitored $24 \mathrm{~h}$ before and for nine days after the influenza challenge. Viral load was measured daily, and self-reported symptoms were collected twice a day. The Random Forest classifier model was used to classify the participants based on changes in the measured parameters. A total of 116 participants with $\sim 3,400,000$ data points were included. Changes in parameters were detected at an early stage of the disease, before the development of symptomatic illness. Heart rate, blood pressure, cardiac output, and systemic vascular resistance showed the greatest changes in the third post-exposure day, correlating with viral load. Applying the classifier model identified participants as flu-positive or negative with an accuracy of $0.81 \pm 0.05$ two days before major symptoms appeared. Cardiac index and diastolic blood pressure were the leading predicting factors when using data from the first and second day. This study suggests that frequent remote monitoring of advanced physiological parameters may provide early pre-symptomatic detection of flu.
\end{abstract}

Keywords: remote patient monitoring; physiological patterns; bio-surveillance; biological outbreak; influenza; photoplethysmography

\section{Introduction}

Influenza (flu) viruses spread easily from person to person by droplet transmission, initiating a quick onset of the disease. Clinically, influenza causes a wide range of clinical signs and symptoms ranging from non-febrile, mild upper-respiratory-tract infection that resolves within up to two weeks to severe or even fatal complications such as pneumonia and sepsis [1]. Influenza patients often exhibit some or all of the following symptoms: fever, chills, cough, sore throat, rhinorrhea, muscle aches, headaches, and fatigue. Influenza can also worsen and complicate asthma, congestive heart failure, pregnancy, morbid obesity, and various other chronic medical conditions [2-6]. Pandemics of influenza can cause 
catastrophic illness and societal disruption and consequently rank high among natural threats that necessitate ongoing public health and medical preparedness [7]. Several influenza pandemics have appeared since the beginning of the 20th Century [8-12] and recently it was shown that a 24-h delay in identification significantly increases the odds of death [6].

It is accepted that vital signs during the acute phase of flu, such as blood oxygen saturation $\left(\mathrm{SpO}_{2}\right)$, heart rate $(\mathrm{HR})$, and respiratory rate $(\mathrm{RR})$ show changes, usually decrease in $\mathrm{SpO}_{2}$, and increase in $\mathrm{HR}$ and $\mathrm{RR}$, yet it is difficult to rely on them as changes are not specific enough, measurement rate is low, and there are no defined patterns characteristic of influenza [13]. Patient characteristics and local surveillance data on influenza outbreaks are important for differential diagnoses and management decisions, owing to differences in clinical outcomes [14]. Traditional bio-surveillance used to detect, inspect, and respond to disease outbreaks has focused on retrospectively gathering and monitoring diagnostic medical and public health data to determine the existence of a disease outbreak [15]. Usually, the information is collected, delivered, and analyzed days, weeks, or even months after the outbreak, and by the time it reaches decision-makers, it may be too late for public health interventions to avoid early cases or to put prevention methods into place.

The distribution of wearable monitors in different settings as an early detection tool, supporting syndromic surveillance during suspected outbreaks, could provide significant advantages and improve timely healthcare delivery $[16,17]$. Moreover, it is postulated that using wearable devices for continuous physiological monitoring could potentially allow prediction and early warning before severe emergencies such as mass biological outbreaks occur [16-18].

This controlled study aimed to monitor healthy subjects who received a novel influenza virus vaccine based on modified vaccinia virus ankara (MVA-NP + M1) using wearable remote patient monitoring devices, looking for potential physiological patterns during the early course of the disease.

\section{Materials and Methods}

\subsection{Study Design and Overview}

This monitoring study was conducted between May 2019 and January 2020 within the framework of a Phase 2, single-center, randomized, double-blinded study evaluating the safety, efficacy, and immunogenicity of a novel flu vaccine in healthy volunteers (NCT03883113). It consisted of an outpatient vaccination phase followed by an inpatient challenge phase two months later. Both the research and the analyzing teams were blinded as to which vaccination was administered to each subject. For the full duration of the study the subjects, including healthy male and female volunteers, were using a wearable wristmonitor (BB-613WP, Biobeat Technologies Ltd., Petah-Tikva, Israel) to collect and record their physiological parameters. The physiological data obtained by the devices were not available to any of the researchers on-site and were analyzed retrospectively. The study was approved by the local Institutional Review Board (Commissie voor Medische Ethiek ZNA, EC Approval Number 5238; the date of registration for the clinical trial was 17 April 2019). All participants signed an informed consent form before the procedure. The study was conducted in a confined research facility (SGS Life Sciences, Clinical Pharmacology Unit, Antwerp, Belgium).

\subsection{Study Procedures and Interventions}

Participants were randomly assigned into vaccine (MVA-NP + M1) or placebo (saline) groups, injected intramuscularly. Two months after intervention they were all challenged intranasally using a Teleflex VaxINator ${ }^{\mathrm{TM}}$ kit with the H3N2 human influenza virus (Figures 1 and 2). 


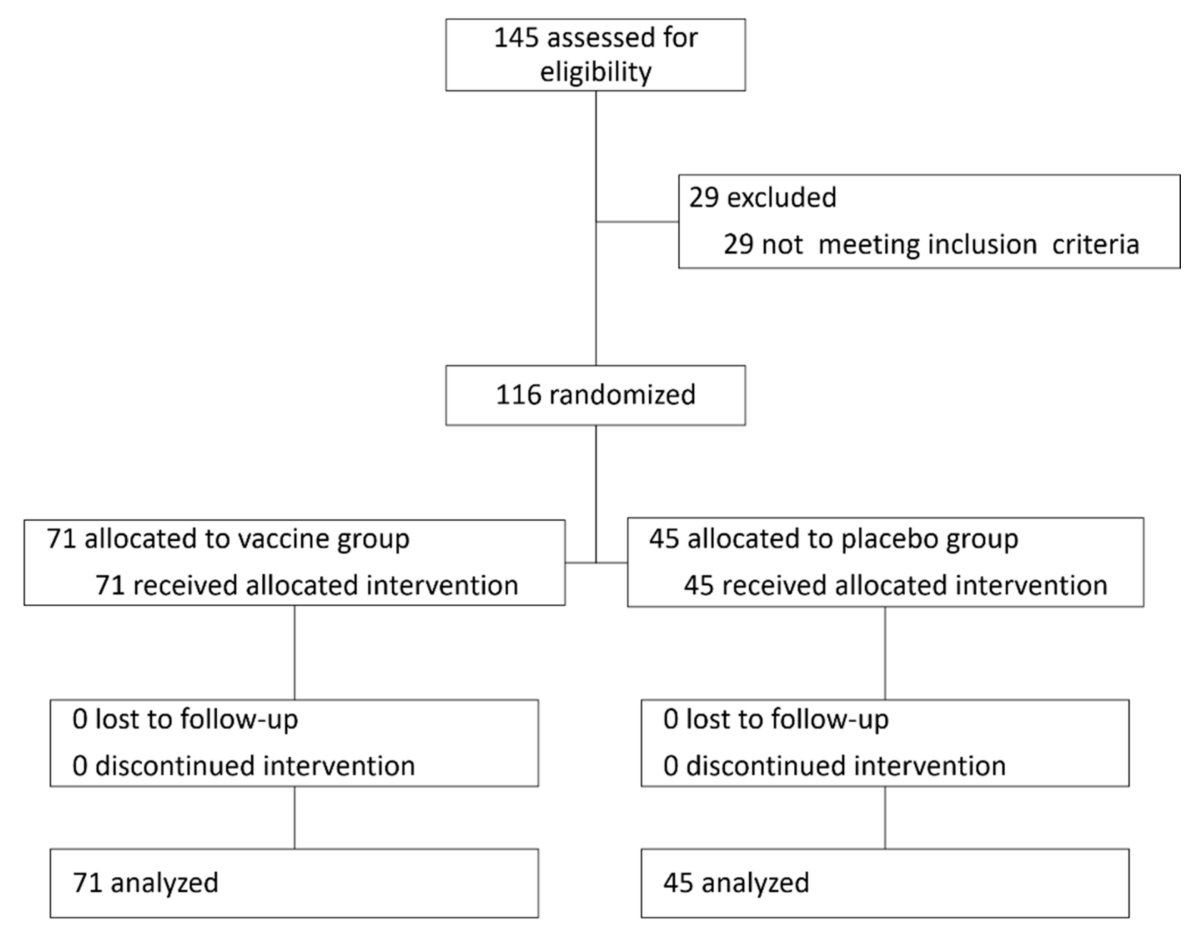

Figure 1. The CONSORT diagram with participants' flow.

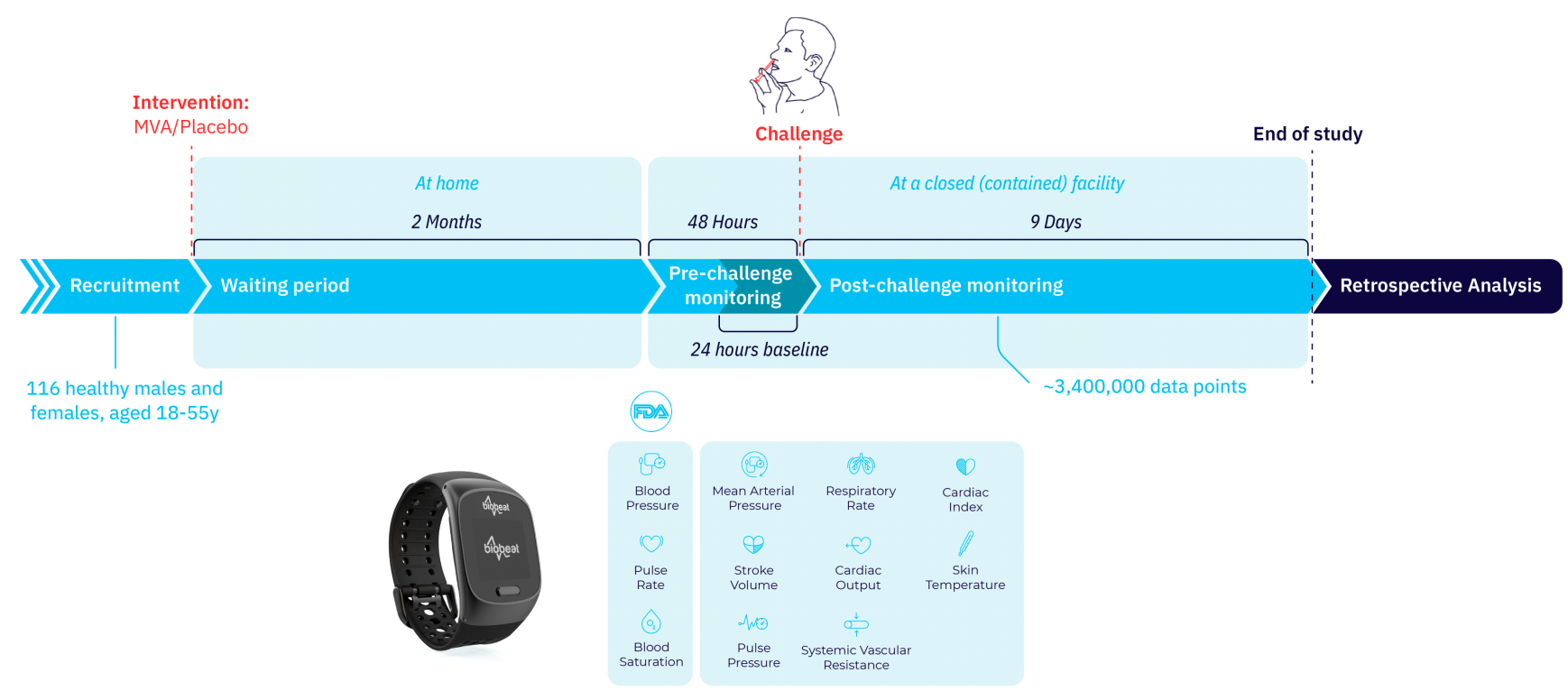

Figure 2. Study design. A total of 116 healthy individuals were recruited. Two months following randomization and treatment with vaccine or placebo, they were admitted into a confined facility in which they were continuously monitored using an FDA-cleared wireless remote patient monitoring device. $48 \mathrm{~h}$ after admission all were intranasally exposed to H3N2 influenza virus, monitoring continued for 9 more days, and daily surveys were completed twice a day during the whole 11 days. The physiological parameters were collected automatically with no personally identifiable information and analyzed retrospectively following study completion.

Continuous monitoring of 12 physiological parameters started $24-48 \mathrm{~h}$ prior to virus inoculation and during the following 9 days. For all measured parameters, the baseline period was defined as the mean value for each participant during the $24 \mathrm{~h}$ before exposure. Physiological parameters were automatically measured for each subject every 5 min throughout the whole study period using the wrist-monitors. These parameters included $\mathrm{HR}, \mathrm{SpO}_{2}, \mathrm{RR}$, systolic blood pressure (SBP), diastolic blood pressure (DBP), mean arterial 
pressure (MAP), pulse pressure (PP), stroke volume (SV), cardiac output (CO), cardiac index (CI), systemic vascular resistance (SVR), and temperature. The collected data had no personal identifiable information (PII) besides serial numbers of the devices.

\subsection{The Wearable Monitoring Devices}

The medical-grade monitoring devices used in the study employ reflective photoplethysmography (PPG) technology, capturing changes in tissue reflectance and measuring numerous vital signs including advanced hemodynamic parameters [19-23]. More specifically, the waveform is related to the reflection caused by the blood vessels when they change diameter. The high resolution of the PPG wave alongside advanced algorithms analyze the pulse wave transit time (PWTT) and provide pulse wave analysis (PWA) which allows the sensor to track vital signs as well as to capture changes, derived from the pulse delineations. Data are transmitted through Bluetooth low energy (BLE) to a dedicated gateway, and from the gateway, the data is transmitted through either $\mathrm{Wi}-\mathrm{Fi}$, cellular, or ethernet to a cloud-based repository, for final analysis.

The wrist monitors require a single trimonthly calibration of the pulse rate (PR) and blood pressure (BP) baseline using an approved non-invasive cuff-based device. Within the context of this study, calibration was performed only once at the beginning of the monitoring period. The wrist monitor is FDA-cleared for heart rate, $\mathrm{SpO}_{2}$, and noninvasive cuffless BP measurements and has a CE mark approval for all vitals. In this study, all vitals were monitored and included in the analysis. The calibration process starts with the individual sitting at rest, as defined by international guidelines on blood pressure measurement. After assuring the cuff size is appropriate for the individual's arm, a measurement is taken using an approved cuff-based device. This is repeated three times, and the average of the three measurements is entered as the baseline calibration value. This is the same for the heart rate value. The temperature is measured using the "double-sensor" technique with thermistors included within the sensor unit.

\subsection{Laboratory Tests}

Nasal swabs for influenza virus were collected twice a day (at least $8 \mathrm{~h}$ apart) from day 2 to day 10 following the intranasal challenge, tested for influenza, PCR, and culture. Viral load was measured by qPCR and virus titration assay, with detection threshold defined as $2.18 \log 10$ viral particles/mL for the qPCR and $0.75 \log 10 \mathrm{TCID} 50 / \mathrm{mL}$ for the virus titration assay (defined as the lowest threshold for which these tests can be considered reliable). A positive for influenza was defined with at least two consecutive positive swabs as determined by qPCR and culture, respectively.

\subsection{Daily Questionnaires}

Participants were asked to fill out questionnaires twice a day, in the morning and in the evening. They were filled up to 11 days after the challenge. The questionnaire included questions on medications taken during the last $12 \mathrm{~h}$, presence of lymphadenopathy, whether they feel well enough to work, whether there are signs of upper respiratory tract infection (including nasal congestion, runny nose, sneezing and its frequency, sinus pressure, or facial pain, sore throat, difficulty swallowing, teary or watery eyes, painful eyes (aversion to light), whether there are signs of lower respiratory tract infection (dry cough, productive cough, difficulty in breathing), gastrointestinal complaints (nausea, stomach ache, vomiting, diarrhea), and general complaints (malaise, dizziness, head congestion, headache, muscle or joint aches, fever, chills, shivering, lack of appetite, feeling cold, sweating, tiredness or weakness, sleeping more than usual, fatigue, or itching). 


\subsection{Statistical Analysis}

All data are presented as mean \pm standard deviation. Between-groups comparison was carried out using independent samples $t$-test. Special attention was given to subgroups of sex and age, looking for a potential difference in the physiological response to flu between males and females, as well as between different age groups. For the comparison of changes in the physiological parameters over time, we used mixed-model ANOVA to evaluate time-treatment, time-sex, time-age, and time-detected virus interactions. For between-days comparison, we used repeated-measures ANOVA, and in cases where significant difference was achieved, Tukey's posthoc test was used. Pearson correlation was used to evaluate the association between changes in the measured parameters and virus levels. Significance was achieved for $p$-value below 0.05 . Data analysis visualizations were made using Pingouin (repeated-measures and mixed ANOVA tests, and for interaction assessment) [24], SciPy stats (independent samples t-test) [25], Scikit-posthocs (Tukey posthoc tests) [26], Scikit-learn (machine learning models) [27], Imblearn (SMOTE) [28], and Seaborn (visualization) [29].

\subsection{Classification Model}

Four classification models (logistic regression, K-nearest neighbors, kernel support vector machine, and random forest classifier) were tested for their ability to classify participants as flu positive or negative, based on the mean daily change from the 24-h baseline period in the measured physiological parameters that were continuouly monitored by the wearable devices. All measured parameters were used as a feature in the model. Due to the imbalanced dataset, the minority class was scaled up by creating synthetic samples using the synthetic minority oversampling technique (SMOTE) method [30] to achieve equal proportions among classes. The data set was stratified into training and validation using repeated K-fold 4 cross-validation (splits $-75 \%$ of the data for training and $25 \%$ for testing, with 3 repeats for a total of 12 combinations) to increase the confidence in the results. SMOTE was used inside the cross-validation process, for each fold. Model performance was evaluated and the following parameters were calculated: recall, precision, f1-score, and the avarage precision, which are more appropitae to imbalanced data [31]. The model with the highest overall score was selected for the calculation and analysis of feature importance.

\section{Results}

In this double-blinded, controlled study, 116 participants (67 females) were included in the analysis out of 145 participants recruited to the vaccine study (see attached Figure 1 with a CONSORT diagram and Figure 2), with a mean age of $40.2 \pm 10.5$ years. 71 participants $(61 \%)$ received the vaccine and 45 received the placebo. No significant differences were observed in baseline characteristics between the treatment groups (Table 1). In both groups, virus levels peaked three days following the challenge and declined from then on (Figure 3A,B). In 11 participants (10 from the MVA group and 1 from the placebo group), virus levels in the routine laboratory tests collected were under the detection threshold, and they were regarded as flu-negative. Within the scope of the vaccine study, the novel flu vaccine did not confer the anticipated protection, with $90.1 \%$ of MVA-treated individuals with positive $\mathrm{qPCR}$ and $77.5 \%$ with a positive culture. 
Table 1. General characteristics of the participants, and average baseline values of the physiological parameters collected during the study. NS, not significant.

\begin{tabular}{|c|c|c|c|c|}
\hline Characteristic & Flu-Positive $(n=105)$ & Flu-Negative $(n=11)$ & $p$-Value & All $(n=116)$ \\
\hline Age (Years) & $39.3 \pm 12.0$ & $40.1 \pm 10.6$ & NS & $40.2 \pm 10.5$ \\
\hline $\operatorname{Sex}(\mathrm{M} / \mathrm{F})$ & $46 / 59$ & $3 / 8$ & NS & $49 / 67$ \\
\hline Respiratory rate (breath/min) & $13.3 \pm 0.7$ & $13.2 \pm 1.2$ & NS & $13.2 \pm 2.3$ \\
\hline $\mathrm{SpO}_{2}(\%)$ & $96.7 \pm 1.2$ & $96.5 \pm 1.5$ & NS & $96.5 \pm 2.9$ \\
\hline Heart rate (beats/min) & $66.2 \pm 8.4$ & $66.6 \pm 7.0$ & NS & $66.6 \pm 12.7$ \\
\hline Systolic blood pressure (mmHg) & $126.0 \pm 10.7$ & $124.4 \pm 9.5$ & NS & $124.7 \pm 11.4$ \\
\hline Diastolic blood pressure (mmHg) & $77.2 \pm 10.6$ & $75.2 \pm 8.4$ & NS & $75.4 \pm 10.1$ \\
\hline Mean arterial pressure $(\mathrm{mmHg})$ & $93.5 \pm 10.1$ & $91.6 \pm 8.2$ & NS & $91.8 \pm 10.0$ \\
\hline Pulse pressure $(\mathrm{mmHg})$ & $48.8 \pm 7.0$ & $49.2 \pm 6.2$ & NS & $49.3 \pm 7.2$ \\
\hline Stroke volume $(\mathrm{mL})$ & $84.3 \pm 10.6$ & $83.3 \pm 11.5$ & NS & $83.1 \pm 13.3$ \\
\hline Cardiac output (L/min) & $5.6 \pm 1.2$ & $5.6 \pm 0.9$ & NS & $5.6 \pm 1.6$ \\
\hline Cardiac index $\left(\mathrm{L} / \mathrm{min} / \mathrm{m}^{2}\right)$ & $3.3 \pm 0.7$ & $3.3 \pm 0.6$ & NS & $3.3 \pm 1.0$ \\
\hline Systemic vascular resistance (dynes $\cdot \mathrm{s} \cdot \mathrm{cm}^{-5}$ ) & $1400.8 \pm 214.0$ & $1411.7 \pm 316.1$ & NS & $1415.2 \pm 388.5$ \\
\hline Temperature $\left({ }^{\circ} \mathrm{C}\right)$ & $36.3 \pm 0.2$ & $36.0 \pm 1.8$ & NS & $36.0 \pm 2.3$ \\
\hline
\end{tabular}

A.

B.
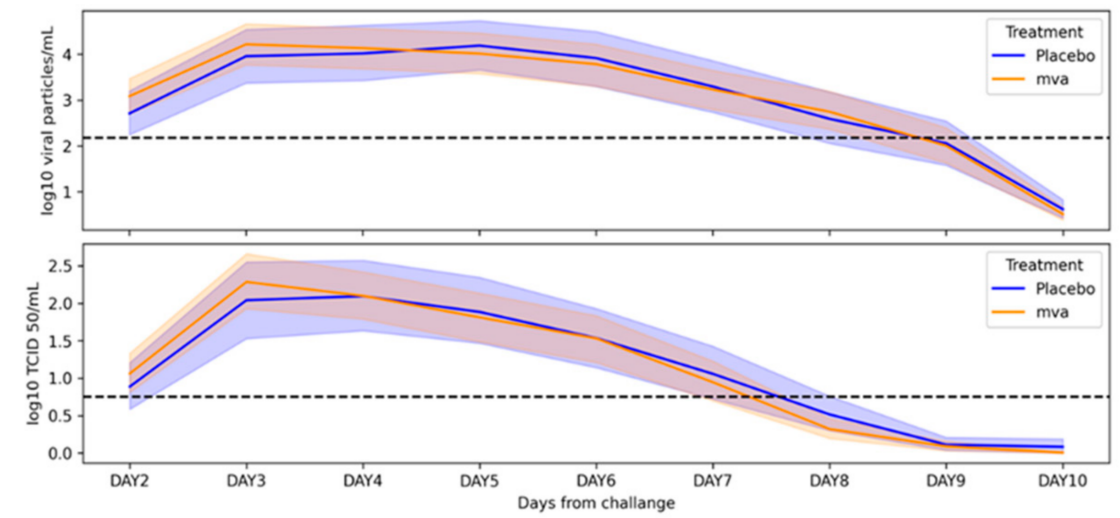

Figure 3. Changes in viral load following exposure among placebo- and MVA-treated groups. Mean $( \pm 95 \%$ confidence interval) viral load as measured by real-time PCR $(\mathbf{A})$ and titration assay (B) throughout the study period. MVA: modified vaccinia virus ankara.

We divided the study cohort into two groups based on the qPCR and the virus titration assay ( $n=105$ positive, $n=11$ negative) and analyzed the mean changes from baseline for all 12 physiological parameters. In parallel to the increase in viral load, the majority of physiological parameters were increased, such as HR $(5.3 \pm 11.9$ beats per minute), SBP $(1.5 \pm 7.4 \mathrm{mmHg}), \mathrm{MAP}(1.3 \pm 5.5 \mathrm{mmHg}), \mathrm{SV}(2.6 \pm 7.5 \mathrm{~mL})$, and SVR $\left(-128.7 \pm 239.6\right.$ dynes.s.cm $\left.{ }^{-5}\right)$ three days following the infection. This pattern appeared only among participants in the flu-positive group (Figure 4). Interactions were observed in several parameters such as HR, temperature, and cardiac output (Figure 4C,I,L). Among the flu-negative participants, SV was the only parameter that significantly decreased $(-2.3 \pm 4.4 \mathrm{~mL})$ from baseline values. 
A.

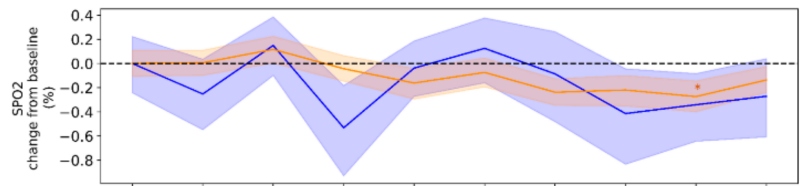

c.

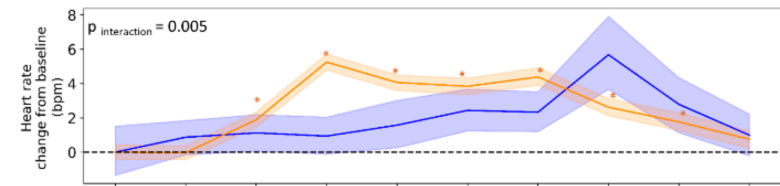

E.

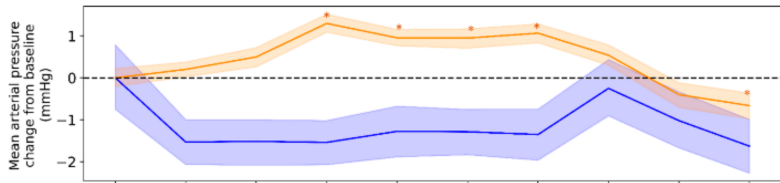

G.

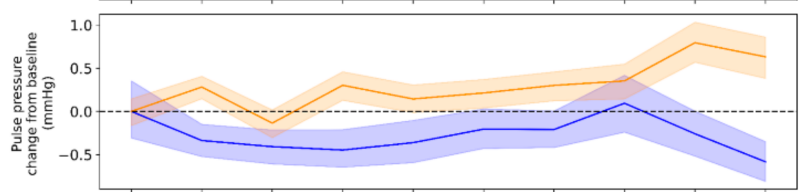

I.

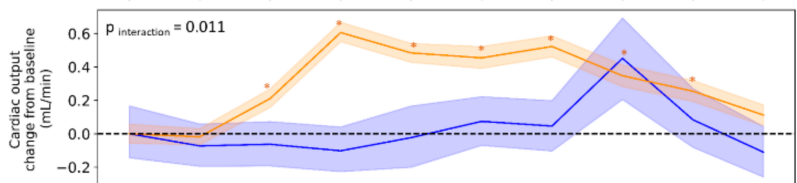

K. 。ั

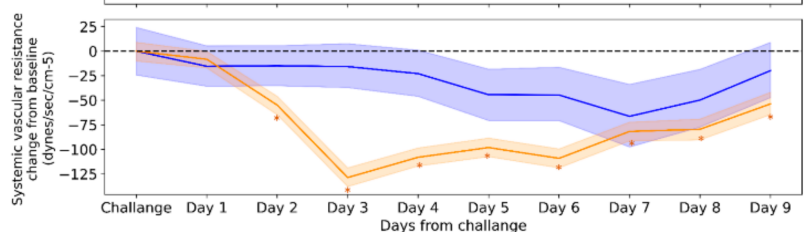

B.

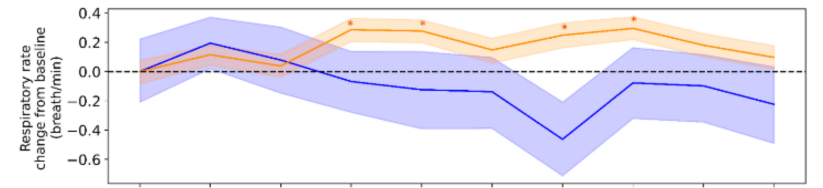

D.

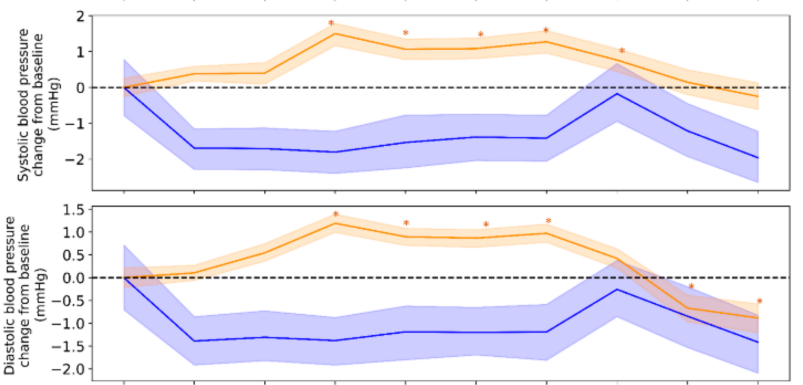

H.

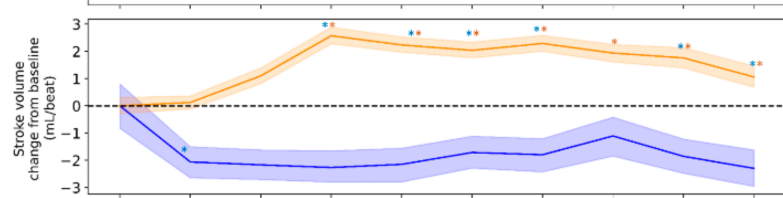

J.

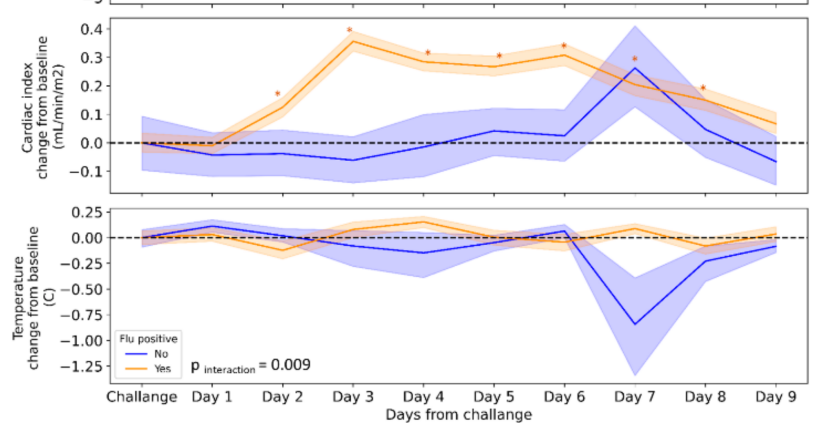

Figure 4. Changes in physiological parameters following exposure among participants in which virus was detected and among participants in which virus was not detected. Relative changes from baseline in physiological parameters (A-L) among participants in which virus was detected (blue lines) or not detected (orange lines). Dashed line represents the baseline. The interaction was assessed using mixed-model ANOVA. * Value significantly $(p<0.05)$ different from baseline.

Next, we looked at whether the changes in the measured parameters were associated with changes in the viral load tests. We found that for the entire population, the highest correlations were achieved on the third day between RT-PCR results and changes in HR $(r=0.516), \mathrm{CO}(r=0.482)$, and $\mathrm{RR}(r=0.286), p<0.001$ for all. We therefore analyzed the correlations on the third day among the different groups-treatment (placebo vs. MVA), sex, and age (Figure 5). Similar trends were observed between placebo- and vaccine-treated participants (Figure 5A). In addition, stronger correlations were achieved between RTPCR results and changes in HR ( $r=0.551$ and 0.450), CO $(r=0.517$ and 0.429$)$, and SVR $(r=-0.601$ and -0.495$)$ in females and in older participants (Figure 5B,C, respectively), while among males and young participants, a stronger association $(r=0.348$ and 0.341$)$ was achieved with changes in RR, respectively.

Finally, we analyzed pre-symptomatic physiological changes in all measured parameters among all participants using four classification models: logistic regression, k-nearest neighbors, kernel support vector machine and random forest. This analysis aimed to define the physiological pattern of changes characterizing this pre-symptomatic phase of flu, to help in the development of a future predictive tool which might be used for bio-surveillance. 

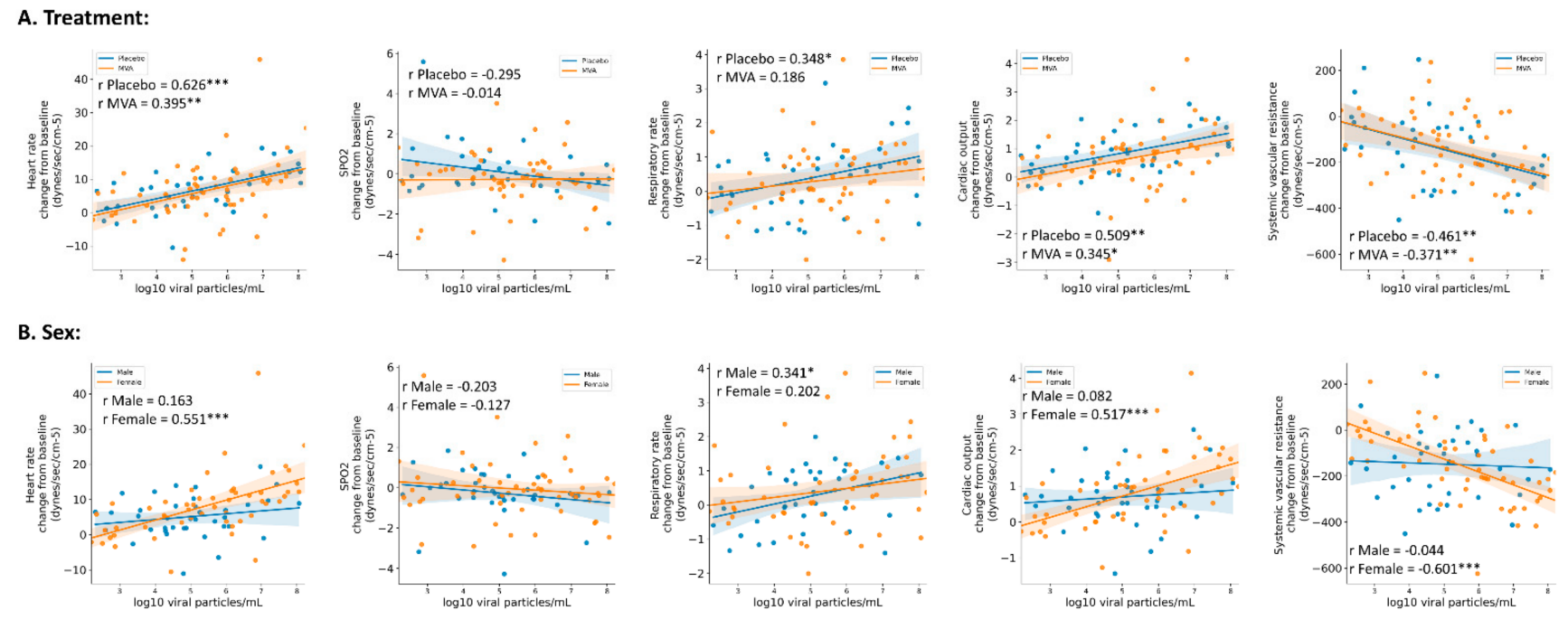

\section{Age group:}
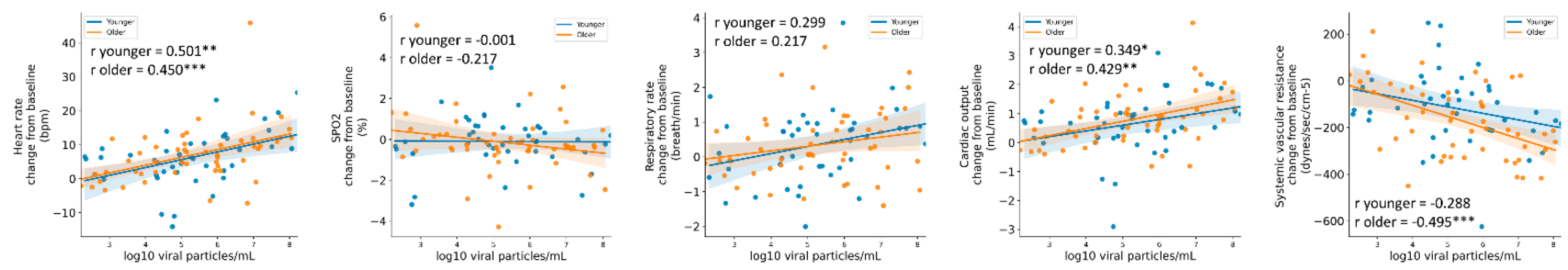

Figure 5. Correlations between viral load and changes in physiological parameters during the third-day post-exposure. Pearson's correlations between viral loads as measured by real-time PCR and mean changes in physiological parameters from the third-day post-exposure. The correlations were stratified based on treatment ((A) Placebo, blue dots/line; MVA, orange dots/line), sex ((B) male, blue dots/line; female, orange dots/line), and age ((C) younger, blue dots/line; older, orange dots/line). ${ }^{*} p<0.05,{ }^{* *} p<0.01,{ }^{* * *} p<0.001$.

According to the lab tests and survey results, we defined the third day as the day in which the disease was most definite in terms of severity (the combination of higher viral load together with complaints). Therefore, we tested whether we can use the measured physiological parameters in the first and second days after exposure to classify the participants as flu-positive or negative before any major symptoms appear (trying to define changes during the pre-symptomatic phase). Accordingly, mean changes from baseline measurements were calculated for each participant, for both days and used as features in the model. Since the data is imbalanced $(n=11$ and 105 for asymptomatic and symptomatic participants, respectively), we used a common method (SMOTE, see methods) to randomly scale up the asymptomatic cases. Among the four models that were tested (logistic regression, k-nearest neighbors, kernel support vector machine, and random forest), random forest achieved the highest overall score (Figure 6A). Using random forest classification, we were able to classify participants as flu-positive or negative with an F1 score (the harmonic mean of precision and recall) of $0.89 \pm 0.04$ and $0.89 \pm 0.03$ by using data from the first and second days, respectively. Avarage precision (AP, weighted mean of precision-recall curve) was slightly greater when using data from the second day $(0.91 \pm 0.04)$ (Figure 6A). Cardiac index and diastolic blood pressure had the greatest importance when using data from the first ( 0.155 and 0.134 , respectively) and second day ( 0.126 and 0.142 , respectively) (Figure 6B), with both found as the best predictors of disease onset. 
A.

\begin{tabular}{|c|c|c|c|c|}
\hline & Recall & Precision & F1 score & AP \\
\hline \multicolumn{5}{|c|}{ Logistic regression } \\
\hline Day 1 & $0.63 \pm 0.06$ & $0.91 \pm 0.04$ & $0.75 \pm 0.05$ & $0.91 \pm 0.03$ \\
\hline Day 2 & $0.59 \pm 0.1$ & $0.89 \pm 0.04$ & $0.70 \pm 0.08$ & $0.90 \pm 0.02$ \\
\hline \multicolumn{5}{|c|}{ K-nearest neighbors } \\
\hline Day 1 & $0.68 \pm 0.08$ & $0.92 \pm 0.03$ & $0.77 \pm 0.05$ & $0.91 \pm 0.02$ \\
\hline Day 2 & $0.64 \pm 0.07$ & $0.89 \pm 0.03$ & $0.74 \pm 0.05$ & $0.89 \pm 0.02$ \\
\hline \multicolumn{5}{|c|}{ Kernel support vector machine } \\
\hline Day 1 & $0.72 \pm 0.10$ & $0.91 \pm 0.02$ & $0.80 \pm 0.06$ & $0.91 \pm 0.02$ \\
\hline Day 2 & $0.71 \pm 0.12$ & $0.89 \pm 0.03$ & $0.78 \pm 0.08$ & $0.89 \pm 0.02$ \\
\hline \multicolumn{5}{|c|}{ Random Forest classifier } \\
\hline Day 1 & $0.89 \pm 0.06$ & $0.90 \pm 0.03$ & $0.89 \pm 0.04$ & $0.90 \pm 0.03$ \\
\hline Day 2 & $0.87 \pm 0.07$ & $0.91 \pm 0.05$ & $0.89 \pm 0.03$ & $0.91 \pm 0.04$ \\
\hline
\end{tabular}

B.
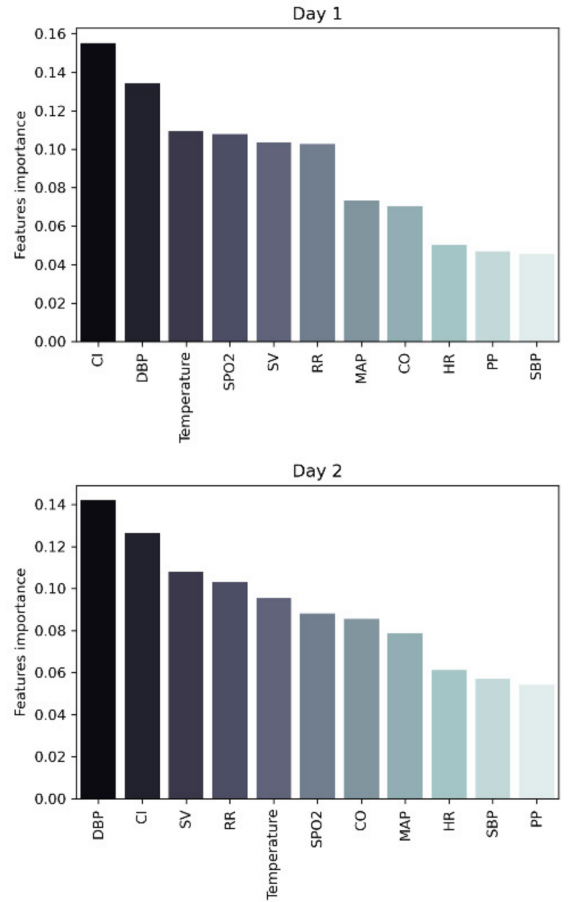

Figure 6. Classification models used for the prediction of viral infection based on changes in physiological parameters. Four models were tested for viral infection classification: logistic regression, k-nearest neighbors, kernel support vector machine, and random forest. Models' performance was assessed using several metrices (A). Panel (B) features importance as calculated for the random forest model in days one and two before infection. Data are presented as mean \pm standard deviation. DBP: diastolic blood pressure; CI: cardiac index; SV: stroke volume; RR: respiratory rate; $\mathrm{SPO}_{2}$ : blood oxygen saturation; CO: cardiac output; MAP: mean arterial pressure; HR: heart rate; SBP: systolic blood pressure; PP: pulse pressure.

\section{Discussion}

In this study, we show that frequent monitoring using the wearable remote monitoring platform may be used in clinical studies by providing advanced clinical insights previously not available. Moreover, collection of multiple physiological parameters is of clinical importance, not only within the scope of clinical studies but also in the daily lives of individuals. As early detection of a biological outbreak is a major mission of national health systems worldwide, as recently highlighted in the COVID-19 pandemic, this tool could allow for improved response due to its automatic, continuous, and real-time collection and transmission of objective physiological data. Currently-used bio-surveillance systems provide important epidemiological data. However, it is still difficult to rely on this data for early situational awareness as this data is acquired in hindsight and usually, after an outbreak of a disease is in advanced stages. In many cases, it includes mainly subjective information based on questionnaires completed by participants.

We assumed that following treatment with the vaccine or the placebo, we will have two distinct groups of participants. Since the novel H3N2 vaccine did not confer protection against the virus, the results did not show such a distinct difference between the two groups.

When comparing the MVA-treated to the placebo-treated individuals, we see that with most physiological parameters, there were no differences between the groups. Differences were seen in diastolic blood pressure and in mean arterial pressure, with the placebotreated group showing an increase in these vitals when compared to the baseline levels. Since the vaccine was not effective, we cannot tell whether this observation is the result of the effect of the vaccine.

Importantly, though no time-symptom interactions were found, and similar trends were observed in both groups, the majority of changes reached significance only among participants in the symptomatic group. 
$\mathrm{SpO}_{2}, \mathrm{RR}, \mathrm{HR}$, and temperature are regarded as relevant vital signs in the case of influenza. However, it is well accepted that they are not sensitive enough to allow detection, especially during the early phase of the infection [13]. We show here that a set of advanced physiological parameters, some of which were not monitored in previous studies due to technological limitations, could provide an early warning of infection. The elevation in $\mathrm{SV}, \mathrm{CO}$, and $\mathrm{CI}$ along with the reduction in SVR following the inoculation might provide additional insights in our understanding of the natural history of flu.

As mentioned, most of the physiological changes appeared in the cardiovascular system, and did not correlate with the severity of the upper respiratory tract complaints mentioned by the participants. In addition, while no changes or minor changes (up to $0.3 \%$ ) were observed in $\mathrm{SpO}_{2}$ and $\mathrm{RR}$, changes in advanced cardiovascular parameters ( $\mathrm{HR}, 59 \%$; SBP, $1.5 \%$; DBP, $2 \%$; MAP, $2 \%$; SV, $4 \%$; CO, $10 \%$; CI, $12 \%$; SVR, $10 \%$ ) were apparent before any clinical reports.

By using the Random Forest classifier, we demonstrated how hemodynamic parameters, including DBP, CI, and SV can help distinguish between individuals that will develop full-blown clinical influenza and those that will not show clinical signs and symptoms.

When analyzing the data and comparing between individuals in which the virus was detected and individuals in which the virus was not detected, we see distinct differences, strengthening the notion that changes are more related to the viral load than to the treatment provided.

Physiological changes started to appear before the individuals had complaints and symptoms, and reached their peak on the third day after exposure. The peak of physiological changes appeared when the subjects only started to complain and show symptoms, and with a concurrent increase in viral load, emphasizing that these processes appear at the same time and show high correlation. This is a strong indication that early-stage monitoring may potentially serve as an early indicator of infection, improve situational awareness, and allow for early use of interventional measures such as isolation and medical treatment.

We found differences in the extent of physiological changes among the groups, with the middle age group showing increased changes when compared to the young, and males showing increased changes when compared to women. This might have significance when trying to better define the natural history of influenza infection in humans, especially concerning early detection and treatment adjustments.

Defining a pattern of change among these physiological parameters might enable us to predict and detect early influenza infections in the future, thus helping health care systems to battle a potential pandemic.

\section{Limitations}

Since the vaccine was found to be ineffective, the majority of participants were flu-positive, which affected the ability of classification models to identify flu-negative participants.

These results from high numbers of flu-positive participants vs. flu-negative limits direct adoption to real-world scenarios. Further balanced studies could allow better insights into the sensitivity and specificity of this tool. Though even a high number of false positives could still be clinically relevant in the case of an outbreak, we should be cautious in this specific study, since we had a small number of participants, especially flu-negative.

The cohort included in this study was limited in size and in individuals' characterizations. A larger cohort with a wider range of age and BMI is needed to substantiate the results, improve our understanding of the physiological changes seen before and during influenza, and better represent the US population. Moreover, this was not a field study, rather a well-controlled trial within a confined facility to assure flu would not spread, making it difficult to simulate a real-world scenario. In contrast, it was easier to perform the exposure procedure and collect the data, showing that prolonged monitoring of subjects using a remote patient monitoring platform is feasible. Therefore, the results could serve as a starting point for future studies of this topic, with additional research in more balanced 
classes in all mentioned aspects. This could ultimately enable a better performance of the model and provide better tools for early pre-symptomatic detection of flu.

As more and more individuals are using wearable devices in the everyday life, and the quality of these devices, as well as the number of variables measured, keeps increasing, it could be estimated that in the future, more people will be using such devices that could help with early detection of outbreaks among the general public [32].

Similar studies would allow us to better differentiate the patterns we found with flu, from other causes of disease, improving its applicability in real-world settings.

\section{Conclusions}

Continuously monitoring advanced hemodynamic parameters could help in defining early pre-symptomatic changes during the early stages of influenza. This has the potential to improve future bio-surveillance efforts by providing early detection of outbreaks, improving situational awareness, and enabling better use of isolation measures and medical treatments.

Author Contributions: Conceptualization, A.E., C.J.A. and G.J.Y.; methodology, N.G., A.E., E.S., R.L., D.N. and Y.G.; software, N.G. and A.B.I.; validation, A.E. and Y.G.; formal analysis, N.G., A.E., C.J.A., E.S., A.B.I., R.M., M.F., D.N. and Y.G., investigation, N.G., A.E., C.J.A., A.B.I. and Y.G.; resources, A.E., G.J.Y. and A.B.I.; data curation, A.E., A.B.I., R.M., M.F., R.L. and Y.G.; writingoriginal draft preparation, N.G., A.E., R.L. and Y.G.; writing-review and editing, N.G., A.E., C.J.A., G.J.Y., E.S., A.B.I., R.M., M.F., R.L., D.N. and Y.G.; visualization, N.G., A.E., C.J.A., G.J.Y., E.S. and Y.G.; supervision, A.E. and A.B.I.; project administration, A.E., C.J.A., G.J.Y. and A.B.I., funding acquisition, A.E. and G.J.Y. All authors have read and agreed to the published version of the manuscript.

Funding: This research was funded by DHHS, grant number 75A50119C00035.

Institutional Review Board Statement: The study was conducted according to the guidelines of the Declaration of Helsinki, and approved by the Institutional Review Board of ZNA, EC Approval Number 5238; date of registration for the clinical trial was 17 April 2019.

Informed Consent Statement: Informed consent was obtained from all subjects involved in the study.

Data Availability Statement: The data that support the findings of this study are available on request from the corresponding author (A.E.). The data are not publicly available due to definitions of the funding authority.

Acknowledgments: We would like to thank the Health \& Human Services, Biomedical Advanced Research and Development Authority for their help and collaboration.

Conflicts of Interest: Authors N.G., A.E., E.S., A.B.I., R.M., M.F. and R.L. are employed by Biobeat Technologies Ltd. The remaining authors declare no conflicts of interest. The funders had no role in the design of the study; in the collection, analyses, or interpretation of data; in the writing of the manuscript, or in the decision to publish the results.

\section{References}

1. Writing Committee of the WHO Consultation on Clinical Aspects of Pandemic (H1N1) 2009 Influenza. Clinical Aspects of Pandemic 2009 Influenza A (H1N1) Virus Infection. N. Engl. J. Med. 2010, 362, 1708-1719. [CrossRef] [PubMed]

2. Domínguez-Cherit, G. Critically Ill Patients With 2009 Influenza A(H1N1) in Mexico. JAMA 2009, 302, 1880-1887. [CrossRef]

3. Denholm, J.T.; Gordon, C.L.; Johnson, P.D.; Hewagama, S.S.; Stuart, R.L.; Aboltins, C.; Jeremiah, C.; Knox, J.; Lane, G.P.; Tramontana, A.R.; et al. Hospitalised adult patients with pandemic (H1N1) 2009 influenza in Melbourne, Australia. Med. J. Aust. 2010, 192, 84-86. [CrossRef] [PubMed]

4. Jain, S.; Kamimoto, L.; Bramley, A.M.; Schmitz, A.M.; Benoit, S.R.; Louie, J.; Sugerman, D.E.; Druckenmiller, J.K.; Ritger, K.A.; Chugh, R.; et al. Hospitalized Patients with 2009 H1N1 Influenza in the United States, April-June 2009. N. Engl. J. Med. 2009, 361, 1935-1944. [CrossRef]

5. Louie, J.K.; Acosta, M.; Winter, K.; Jean, C.; Gavali, S.; Schechter, R.; Vugia, D.; Harriman, K.; Matyas, B.; Glaser, C.A.; et al. Factors Associated with Death or Hospitalization Due to Pandemic 2009 Influenza A(H1N1) Infection in California. JAMA 2009, 302, 1896-1902. [CrossRef] 
6. Yang, S.; Zhou, Y.; Cui, Y.; Ding, C.; Wu, J.; Deng, M.; Wang, C.; Lu, X.; Chen, X.; Li, Y.; et al. The need for strengthening the influenza virus detection ability of hospital clinical laboratories: An investigation of the 2009 pandemic. Sci. Rep. 2017, 7, 43433. [CrossRef]

7. Schuchat, A.; Bell, B.P.; Redd, S.C. The Science behind Preparing and Responding to Pandemic Influenza: The Lessons and Limits of Science. Clin. Infect. Dis. 2011, 52, S8-S12. [CrossRef]

8. Taubenberger, J.K.; Reid, A.; Janczewski, T.A.; Fanning, T.G. Integrating historical, clinical and molecular genetic data in order to explain the origin and virulence of the 1918 Spanish influenza virus. Philos. Trans. R. Soc. B Biol. Sci. 2001, 356, 1829-1839. [CrossRef] [PubMed]

9. Outbreak of Swine-Origin Influenza A (H1N1) Virus Infection-Mexico, March-April 2009. Available online: https:/ /www.cdc. gov/mmwr/preview/mmwrhtml/mm5817a5.htm (accessed on 29 June 2021).

10. Swine Influenza A (H1N1) Infection in Two Children-Southern California, March-April 2009. Available online: https://www. cdc.gov/mmwr/preview/mmwrhtml/mm5815a5.htm (accessed on 29 June 2021).

11. Swine-Origin Influenza A (H1N1) Virus Infections in a School-New York City, April 2009. Available online: https://www.cdc gov/mmwr/preview/mmwrhtml/mm5817a6.htm (accessed on 29 June 2021).

12. Fukuyama, S.; Kawaoka, Y. The pathogenesis of influenza virus infections: The contributions of virus and host factors. Curr. Opin. Immunol. 2011, 23, 481-486. [CrossRef]

13. Simonsen, L.; Spreeuwenberg, P.; Lustig, R.; Taylor, R.J.; Fleming, D.M.; Kroneman, M.; Van Kerkhove, M.D.; Mounts, A.W.; Paget, W.J.; the GLaMOR Collaborating Teams. Global Mortality Estimates for the 2009 Influenza Pandemic from the GLaMOR Project: A Modeling Study. PLoS Med. 2013, 10, e1001558. [CrossRef]

14. Lee, N.; Chan, P.; Lui, C.Y.G.; Wong, B.C.K.; Sin, W.W.Y.; Choi, K.-W.; Wong, R.Y.K.; Lee, E.L.Y.; Yeung, A.C.M.; Ngai, K.L.K.; et al. Complications and Outcomes of Pandemic 2009 Influenza A (H1N1) Virus Infection in Hospitalized Adults: How Do They Differ from Those in Seasonal Influenza? J. Infect. Dis. 2011, 203, 1739-1747. [CrossRef] [PubMed]

15. Shmueli, G.; Burkom, H. Statistical Challenges Facing Early Outbreak Detection in Biosurveillance. Technometrics 2010, 52, 39-51. [CrossRef]

16. Ming, D.K.; Sangkaew, S.; Chanh, H.Q.; Nhat, P.T.; Yacoub, S.; Georgiou, P.; Holmes, A.H. Continuous physiological monitoring using wearable technology to inform individual management of infectious diseases, public health and outbreak responses. Int. J. Infect. Dis. 2020, 96, 648-654. [CrossRef]

17. Radin, J.M.; Wineinger, N.E.; Topol, E.J.; Steinhubl, S.R. Harnessing wearable device data to improve state-level real-time surveillance of influenza-like illness in the USA: A population-based study. Lancet Digit. Health 2020, 2, e85-e93. [CrossRef]

18. Seshadri, D.R.; Davies, E.V.; Harlow, E.R.; Hsu, J.J.; Knighton, S.C.; Walker, T.A.; Voos, J.E.; Drummond, C.K. Wearable Sensors for COVID-19: A Call to Action to Harness Our Digital Infrastructure for Remote Patient Monitoring and Virtual Assessments. Front. Digit. Health 2020, 2, 8. [CrossRef]

19. Nachman, D.; Gilan, A.; Goldstein, N.; Constantini, K.; Littman, R.; Eisenkraft, A.; Grossman, E.; Gepner, Y. 24-Hour Ambulatory Blood Pressure Measurement Using a Novel Noninvasive, Cuffless, Wireless Device. Am. J. Hypertens. 2021. [CrossRef]

20. Nachman, D.; Gepner, Y.; Goldstein, N.; Kabakov, E.; Ben Ishay, A.; Littman, R.; Azmon, Y.; Jaffe, E.; Eisenkraft, A. Comparing blood pressure measurements between a photoplethysmography-based and a standard cuff-based manometry device. Sci. Rep. 2020, 10, 16116. [CrossRef] [PubMed]

21. Atzmon, Y.; Ben Ishay, E.; Hallak, M.; Littman, R.; Eisenkraft, A.; Gabbay-Benziv, R. Continuous Maternal Hemodynamics Monitoring at Delivery Using a Novel, Noninvasive, Wireless, PPG-Based Sensor. J. Clin. Med. 2020, 10, 8. [CrossRef] [PubMed]

22. Nachman, D.; Constantini, K.; Poris, G.; Wagnert-Avraham, L.; Gertz, S.D.; Littman, R.; Kabakov, E.; Eisenkraft, A.; Gepner, Y. Wireless, non-invasive, wearable device for continuous remote monitoring of hemodynamic parameters in a swine model of controlled hemorrhagic shock. Sci. Rep. 2020, 10, 17684. [CrossRef]

23. Eisenkraft, A.; Maor, Y.; Constantini, K.; Goldstein, N.; Nachman, D.; Levy, R.; Halberthal, M.; Horowitz, N.; Golan, R.; Rosenberg, E.; et al. Trajectories of Key Physiological Parameters in COVID-19 Patients Using Continuous Remote Monitoring and Health AI. Res. Sq. 2020. [CrossRef]

24. Vallat, R. Pingouin: Statistics in Python. J. Open Source Softw. 2018, 3, 1026. [CrossRef]

25. SciPy API-SciPy v1.7.0 Manual. Available online: https://docs.scipy.org/doc/scipy/reference/index.html (accessed on 29 June 2021).

26. Scikit-Posthocs-Scikit-Posthocs 0.6.6 Documentation. Available online: https://scikit-posthocs.readthedocs.io/en/latest/ (accessed on 29 June 2021).

27. Scikit-Learn: Machine Learning in Python—Scikit-Learn 0.24.2 Documentation. Available online: https://scikit-learn.org/stable/ index.html (accessed on 29 June 2021).

28. SMOTE-Version 0.8.0. Available online: https://imbalanced-learn.org/stable/references/generated/imblearn.over_sampling. SMOTE.html (accessed on 29 June 2021).

29. Seaborn: Statistical Data Visualization-Seaborn 0.11.1 Documentation. Available online: https://seaborn.pydata.org/index.html (accessed on 29 June 2021).

30. Chawla, N.V.; Bowyer, K.; Hall, L.O.; Kegelmeyer, W.P. SMOTE: Synthetic Minority Over-Sampling Technique. J. Artif. Intell. Res. 2002, 16, 321-357. [CrossRef] 
31. Saito, T.; Rehmsmeier, M. The Precision-Recall Plot Is More Informative than the ROC Plot When Evaluating Binary Classifiers on Imbalanced Datasets. PLoS ONE 2015, 10, e0118432. [CrossRef]

32. Radin, J.M.; Quer, G.; Jalili, M.; Hamideh, D.; Steinhubl, S.R. The hopes and hazards of using personal health technologies in the diagnosis and prognosis of infections. Lancet Digit. Health 2021, 3, e455-e461. [CrossRef] 